\title{
Initial clinical results and prognostic variables in the implementation of a Sepsis Code in a University Hospital of high complexity level
}

Fernando Ramasco, Angels Figuerola, Rosa Mendez, Ana Hernandez, Andres von Wernitz, Diego Rodriguez and Sepsis Team. Hospital Universitario La Princesa - Madrid (Spain)

Background and Goal of Study:

To know the impact in the first months of the application of a sepsis code in a hospital of high complexity.

Materials and Methods:

A prospective observational study was conducted during a consecutive period of 7 months in 2015.The association between risk factors, severity factors and analytical values with mortality was carried out by univariate and multivariate analysis.

\section{Results and Discussion:}

A total of $\mathbf{2 3 7}$ patients with severe sepsis were included. The average age was 72 years and $60 \%$ were male. $34 \%$ of the patients had some hospital admission in the 3 months prior to the episode of sepsis and $31 \%$ received antibiotherapy in those 3 previous months. $37 \%$ of the cultures were positive, detecting $11 \%$ of multi-resistant germs.

Hospital mortality at 30 days was $24 \%$ and at 60 days, $27 \%$, with the mortality rate of those admitted to the ICU of $30 \%$.

A statistically significant association was found between the administration of more than 2 antibiotics and mortality $(p<0.05)$.

Significant differences were found between the patients who died and the survivors in the mean values of creatinine ( $2.30 \mathrm{vs} 1.46 \mathrm{mg} / \mathrm{dL}$ ), lactic (6.10 vs $2.62 \mathrm{mmol} / \mathrm{L}$ ) and procalcitonin (23.27 vs $12.73 \mathrm{mg} / \mathrm{dL})$.

A statistically significant linear trend was detected between the SOFA scale score and mortality $(\mathrm{p}<0.05)$.

Patients with a shock index $\geq 0.8$ and a lactic $\geq 3 \mathbf{m m o l} / L$ had a 2.5 times higher risk of dying, and in patients with a lactic $\geq 3 \mathrm{mmol} / \mathrm{L}$ and $a$ procalcitonin $\geq \mathbf{2} \mathbf{~} \mathbf{m g} / \mathbf{d L}$ the risk was $\mathbf{8}$ times higher.

In the multivariate analysis on mortality in CSP patients, independent risk factors were identified: age, lactic acid, creatinine and the need for amines

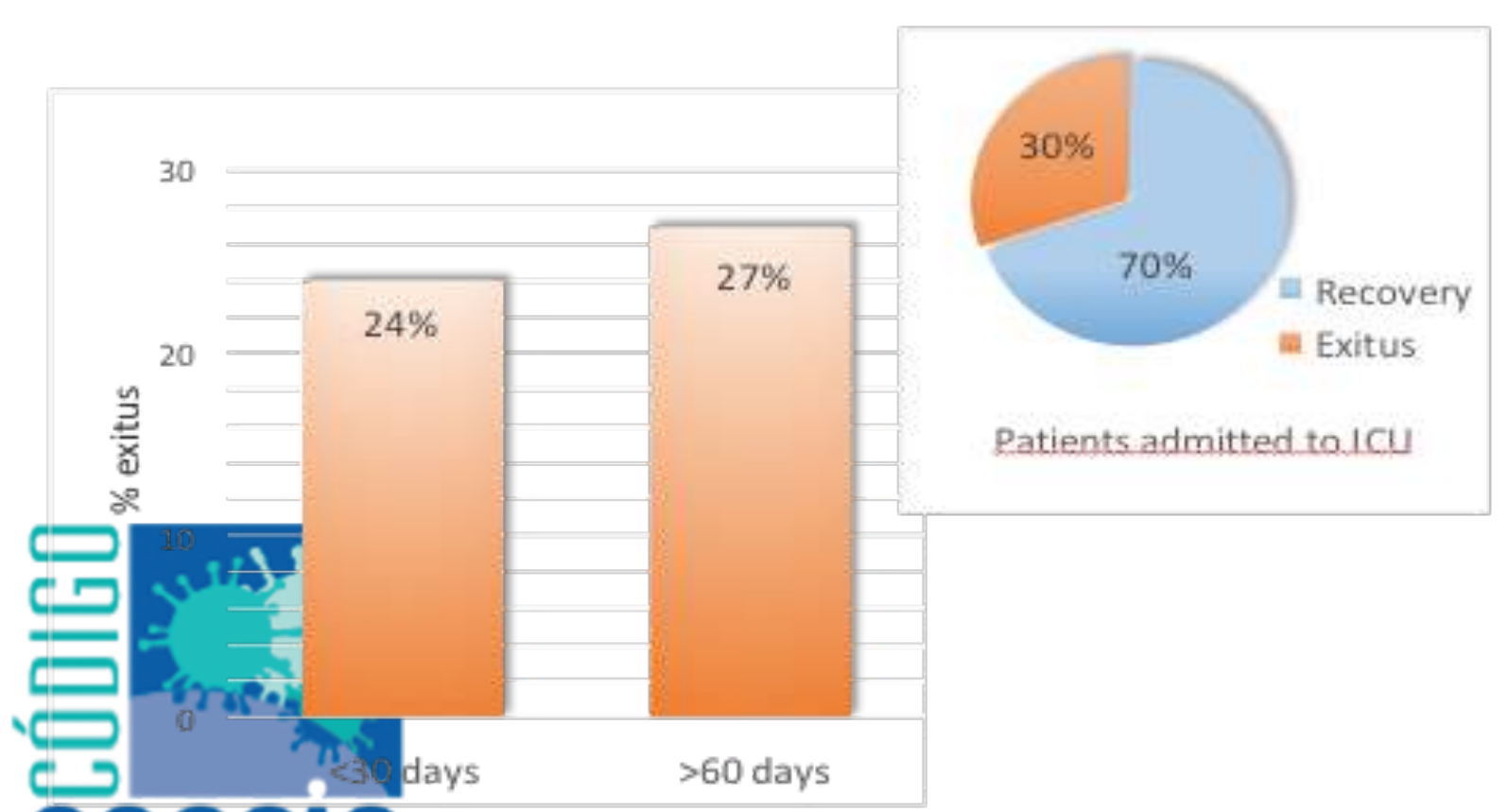

Percentage of exitus in the first 30 days and 60 days after the activation of a Sepsis Code alert and the proportion of exitus

\begin{tabular}{|c|c|c|c|}
\hline & Exitus (N 56) & $\begin{array}{l}\text { No exitus (N } \\
\text { |8I) }\end{array}$ & Significance \\
\hline Heart rate, bpm (SD) & $103(29)$ & $104(23)$ & $p>0.05$ \\
\hline $\begin{array}{l}\text { Systolic blood pressure, } \mathrm{mmHg} \\
\text { (SD) }\end{array}$ & $107(29)$ & $108(28)$ & $p>0.05$ \\
\hline Haematocrit, \% (SD & $36(8.7)$ & 38 (7.I) & $p>0.05$ \\
\hline Leucocytes, miles $/ \mathrm{mm}^{3}$ (SD) & $12.31(10.4)$ & $11.80(13.1)$ & $p>0.05$ \\
\hline Platelets, miles $/ \mathbf{m m}^{3}$ (SD & $210.36(216)$ & $171.81(133)$ & $p>0.05$ \\
\hline Lactic, mmol/L (SD) & $6.10(5.1)$ & $2.62(1.5)$ & $p<0.05$ \\
\hline Procalcitonin, mg/dL (SD & $23.27(32.0)$ & 12.73 (24.2) & $p<0.05$ \\
\hline Creatinine, $\mathrm{mg} / \mathrm{dL}$ (SD) & $2.30(1.4)$ & $1.46(0.9)$ & $p<0.05$ \\
\hline Bilirubin, $\mathbf{m g} / \mathrm{dL}$ (SD) & $1.81(2)$ & $1.09(1)$ & $p>0.05$ \\
\hline
\end{tabular}

Univariate analysis of predictive factors and their association with exitus. SD: Standard Deviation; N: frequency, BPM: Beats per Minute

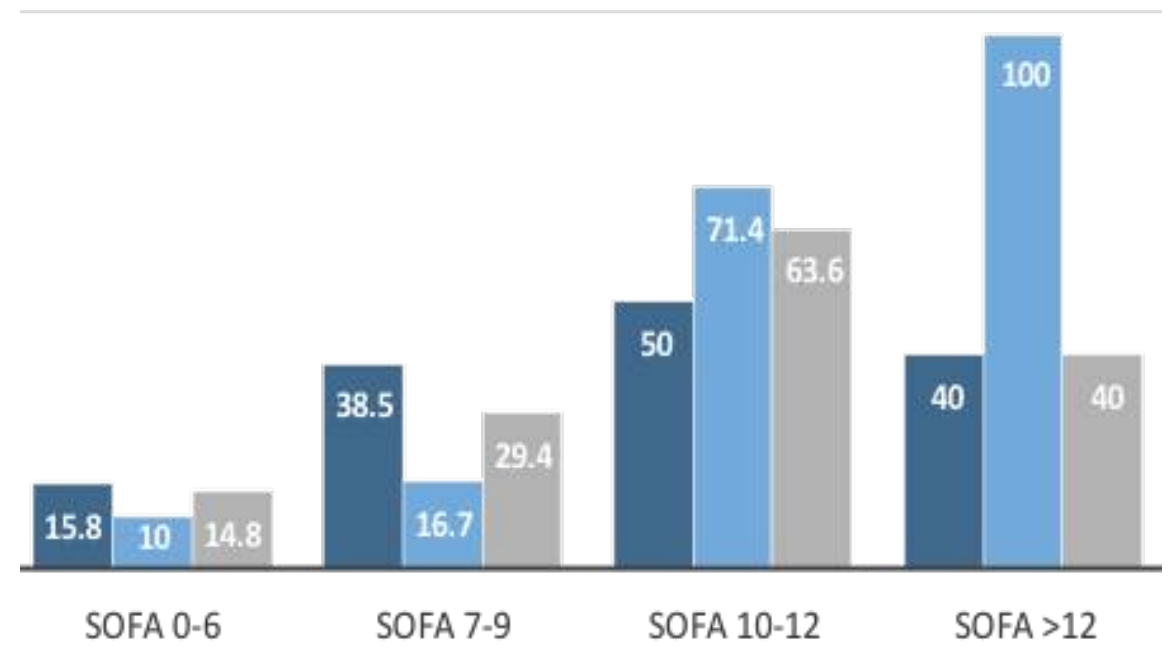

- Activation in Emergency Room $=$ Activation in hospital ward $=$ Admitted to ICU

Percentage of mortality in each group on the SOFA scale, for those patients with activation of Sepsis code Alert in

Conclusion(s):

The epidemiological, clinical and mortality characteristics of the patients in our series after the implementation of the CSP are similar to those published nationally and internationally. Opportunities for improvement have been detected. Indexes and associations of markers have been detected with utility at the local level for risk stratification in patients with sepsis alert. 